\section{Renal involvement at diagnosis of pediatric acute lymphoblastic leukemia}

\author{
Mayerly Prada Rico, ${ }^{1}$ Carmen Inés \\ Rodríguez-Cuellar, ${ }^{2}$ \\ Lucy Natalia Arteaga Aya, ${ }^{3}$ \\ Claudia Lorena Nuñez Chates, ${ }^{4}$ \\ Sandra Patricia Garces Sterling, 5 \\ Mathieu Pierotty, ${ }^{6}$ \\ Luz Esthella González Chaparro, ${ }^{1}$ \\ Ricardo Gastelbondo Amaya1 \\ 1Pediatric Nephrology Division, \\ Pediatrics Deparment, Fundación \\ Cardioinfantil, Bogotá; ${ }^{2}$ Pediatric \\ Nephrology Division, Pediatrics \\ Department, Clínica Shaio, Bogotá; \\ 3Pediatric Deparment, Universidad el \\ Bosque, Bogotá; ${ }^{4}$ Pediatric Deparment, \\ Universidad del Rosario, Bogotá; \\ 5Pediatric Hematology Oncology \\ Division, Pediatrics Department, \\ Fundación Cardioinfantil, Bogotá; \\ 6Radiology and Medical Diagnostic \\ Images, Fundación Cardioinfantil, \\ Bogotá, Colombia
}

\section{Abstract}

Acute leukemia is the most common type of cancer in pediatric patients. This type of cancer accounts for a third of all childhood cancer cases. More than half of pediatric acute leukemia patients show signs and symptoms such as hepatomegaly, splenomegaly, pallor, fever and bruising at the time of diagnosis. In early stages of acute lymphoblastic leukemia (ALL), nephromegaly and other renal manifestations such as high blood pressure (HBP) and renal failure are uncommon, although renal infiltration and nephromegaly are common in advanced-stage pediatric patients. This is a retrospective case review with a critical appraisal of the existing evidence from the literature. We present a clinical case of a child with HBP associated with bilateral nephromegaly which resolved after chemotherapy treatment. This patient presented with HBP that required pharmacological treatment, likely owing to nephromegaly. All HBP secondary causes were rejected. Nephromegaly was resolved after chemotherapy treatment, and antihypertensive medication was discontinued. Nephromegaly and HBP are rare manifestations of ALL debut in pediatrics. The present case report illustrates this unusual combination and Suggests clinicians to consider malignancy as its causal factor, especially if the symptoms are accompanied by other suggestive extrarenal manifestations.

\section{Introduction}

Acute leukemia is the most common type of cancer in pediatric patients, accounting for a third of all cases of childhood cancer. More than half of acute leukemia pediatric patients show signs of hepatomegaly, splenomegaly, pallor, fever and bruising at diagnosis. ${ }^{1}$ During advanced stages of ALL, renal infiltration and nephromegaly are common in pediatric patients. However, nephromegaly and other renal manifestations such as HBP and renal failure are not common at the beginning of the disease. ${ }^{2}$

Nephromegaly can be attributed to leukemic infiltration, hypertrophy or hyperplasia of parenchymal cells. ${ }^{3,4}$ HBP is associated with steroid treatment, ${ }^{2}$ acute renal failure, narrowing or occlusion of intrarenal arteries and leukemic infiltration. ${ }^{5}$ HBP associated with nephromegaly at the time of diagnosis of ALL is usually moderate, transient and does not require pharmacological intervention. ${ }^{2,4,5}$ Here, we report a case of a pediatric patient with nephromegaly and HBP at the time of diagnosis of ALL. With ALL treatment, nephromegaly was resolved, but the patient required pharmacological treatment for hypertension.

\section{Case Report}

A 3-year-old Caucasian boy presented at pediatric emergency with a 3-day history of fever, petechiae on eyelids and pallor. Personal and family history was unremarkable. He was born from a full-term uncomplicated pregnancy with a normal postpartum course.

Physical examination of the patient showed a weight of $16.1 \mathrm{~kg}$, height of 101 $\mathrm{cm}$ and blood pressure of $114 / 76$ ( $>95^{\text {th }}$ percentile). ${ }^{6}$ A complete blood count on the day of admission showed the following results: WBC count $12.300 / \mu \mathrm{L}$ with $86 \%$ blasts, platelets $16.400 / \mu \mathrm{L}$, hemoglobin $7.9 \mathrm{~g} / \mathrm{dL}$. Lactic dehydrogenase was slightly elevated at $383 \mathrm{U} / \mathrm{L}$ (reference value: $85-227 \mathrm{U} / \mathrm{L}$ ), erythrocyte sedimentation rate was 91 $\mathrm{mm} / \mathrm{h}$ (reference value: $0-13 \mathrm{~mm} / \mathrm{h}$ ) and Creactive protein value was $6.67 \mathrm{mg} / \mathrm{dL}$ (reference value: $0-0.3 \mathrm{mg} / \mathrm{dL}$ ).

The rest of the blood chemistry tests were normal (electrolytes, urea nitrogen, serum creatinine, uric acid, bicarbonate, lactate and transaminases). Coagulation tests (prothrombin time and partial throm-
Correspondence: Carmen Rodríguez-Cuellar, Pediatric Nephrologist, Fundación Clínica Shaio, 115a Street 70c, 75, Bogotá, Colombia. Tel.: +573008503346

E-mail: crnefropedia@gmail.com

Key words: Nephromegaly, hypertension, acute lymphoblastic leukemia

Contributions: MP conceived the study, applied for the ethics submission, was involved in all aspects of the paper generation and coordinated all coauthors' activities. LA, CN, wrote the first draft. MP, CR corrected and translated the manuscript, provided critical input and participated in all phases of the paper writing. All authors participated in revising the manuscript critically for important intellectual content and approved the final version to be submitted to the journal.

Conflicts of interest: The authors declare no potential conflict of interest.

Funding: None.

Availability of data and materials: The data that support the findings of this study are available from the corresponding author upon reasonable request.

Ethics approval and consent to participate: This research was conducted in accordance with all relevant guidelines and procedures.

Consent for publication: Consent was obtained.

Received for publication: 18 November 2019.

Revision received: 6 December 2019.

Accepted for publication: 10 December 2019.

This work is licensed under a Creative Commons Attribution NonCommercial 4.0 License (CC BY-NC 4.0).

(C) Copyright: the Author(s), 2020

Licensee PAGEPress, Italy

Pediatric Reports 2020; 12:8382

doi:10.4081/pr.2020.8382

boplastin time) and urinalysis was also normal. Serological tests for cytomegalovirus, human immunodeficiency virus, EpsteinBarr virus and hepatitis $\mathrm{B}$ and $\mathrm{C}$ were negative. Abdominal ultrasound showed enlarged kidneys with normal echo-Doppler findings of renal vessels. Computed tomography (CT) of the abdomen showed kidneys enlarged for age and body surface area, with longitudinal diameter of the right kidney 9.5 $\mathrm{cm}(\mathrm{Z}$ score +4.75$)$ and of the left kidney $9.0 \mathrm{~cm}(Z$ score +3.97$) .{ }^{7}$ Normal corticomedullary differentiation and non-dilated pelvicalyceal system were observed (Figure 1). The liver was also enlarged, with longitudinal diameter of the right hepatic lobe $12.1 \mathrm{~cm}$ (reference value: 6.9-10.9 cm) 

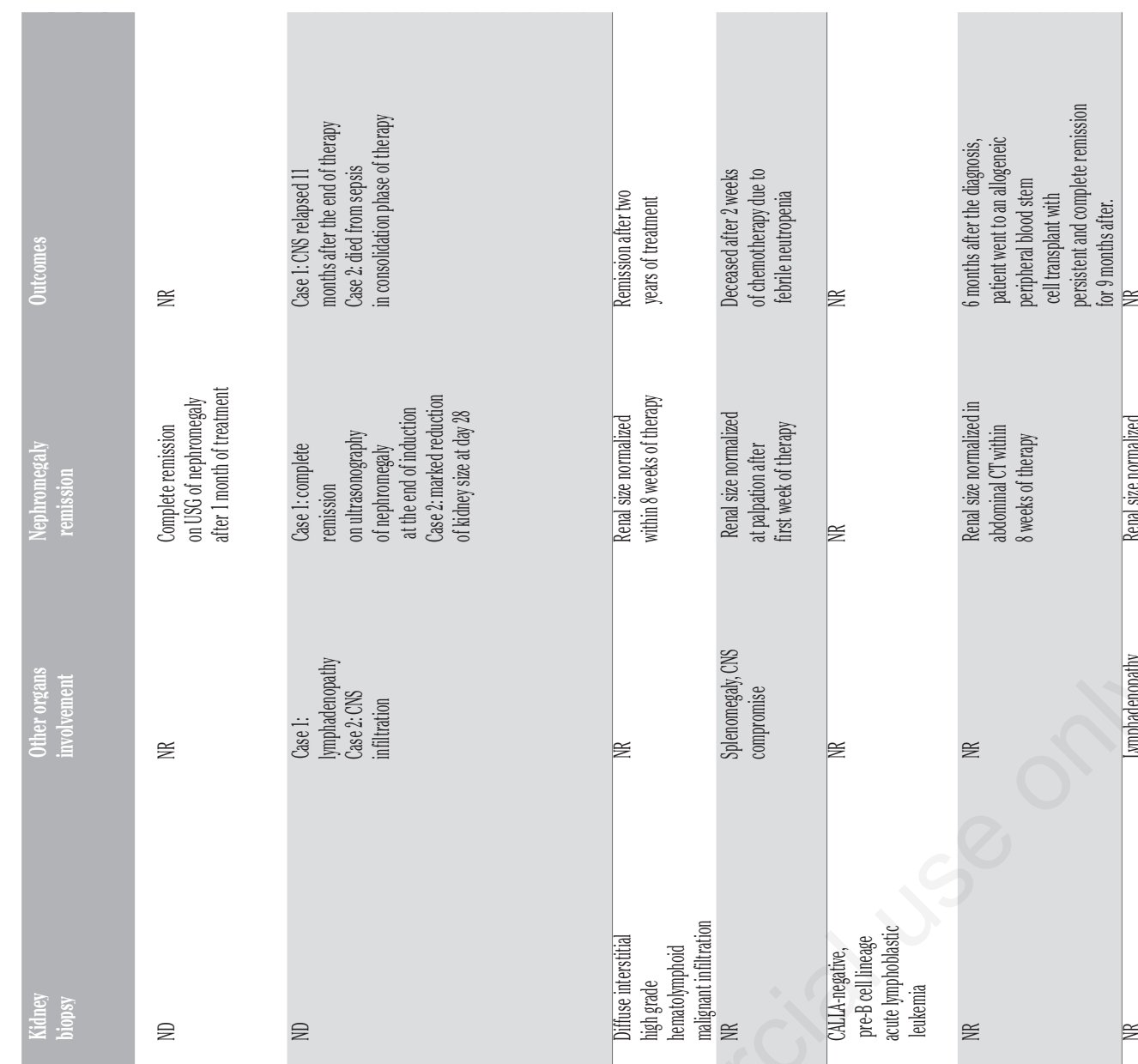

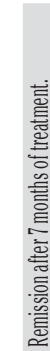

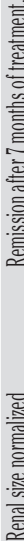

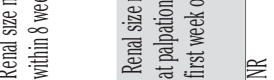
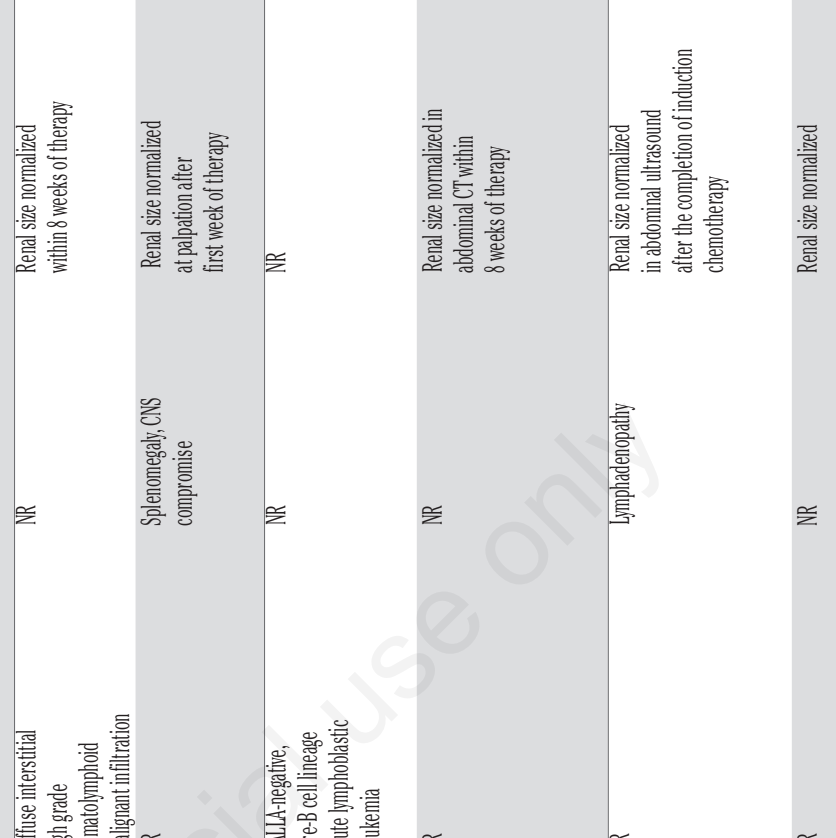

总

总 흥

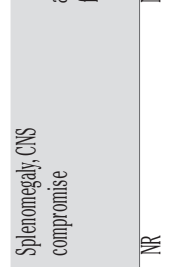

產

$\stackrel{n}{z}$

$\cong$
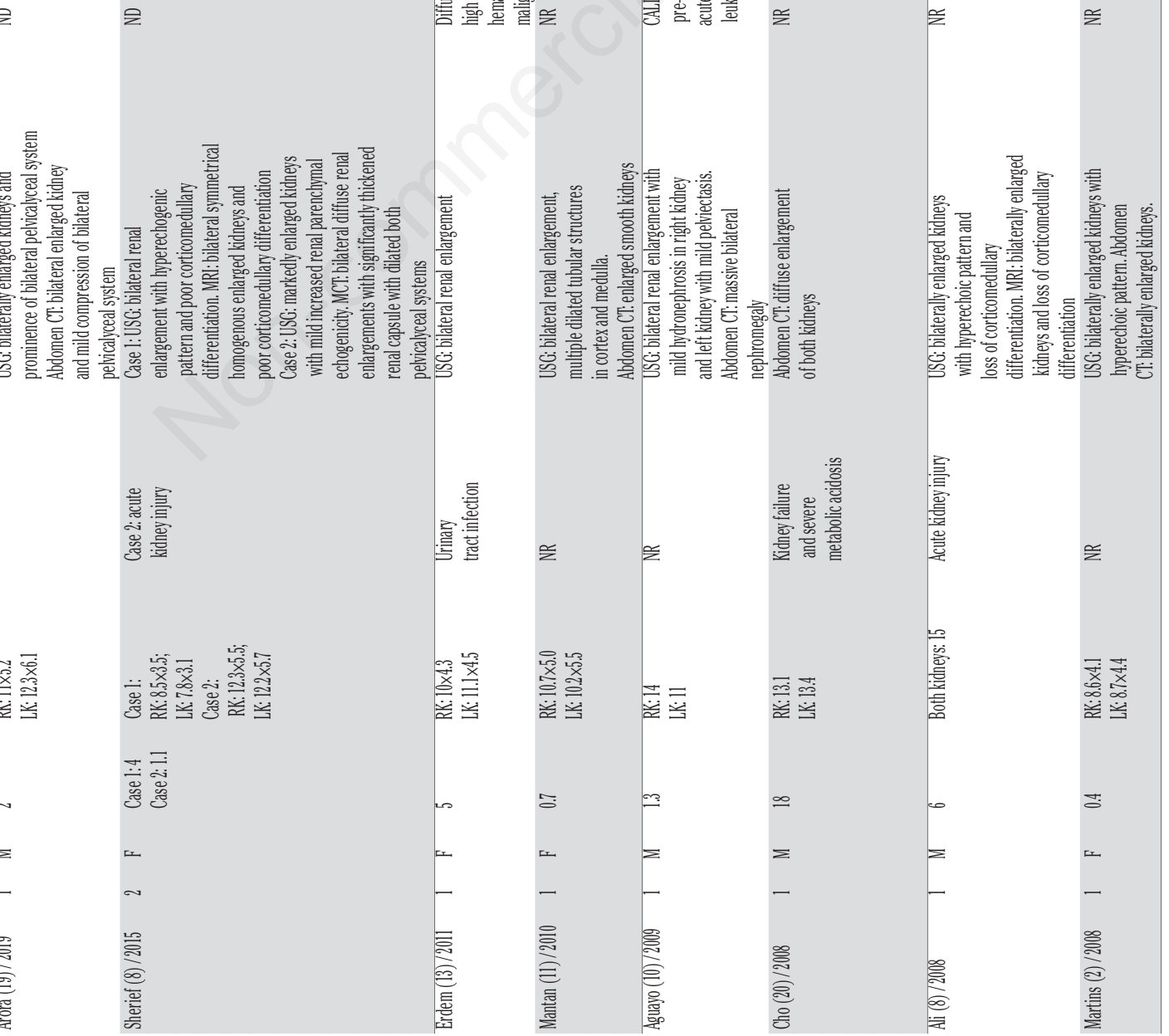

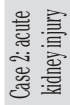

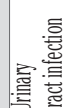

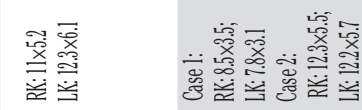

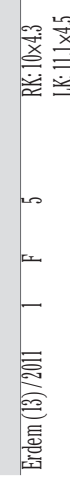

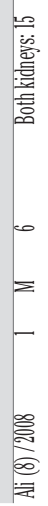




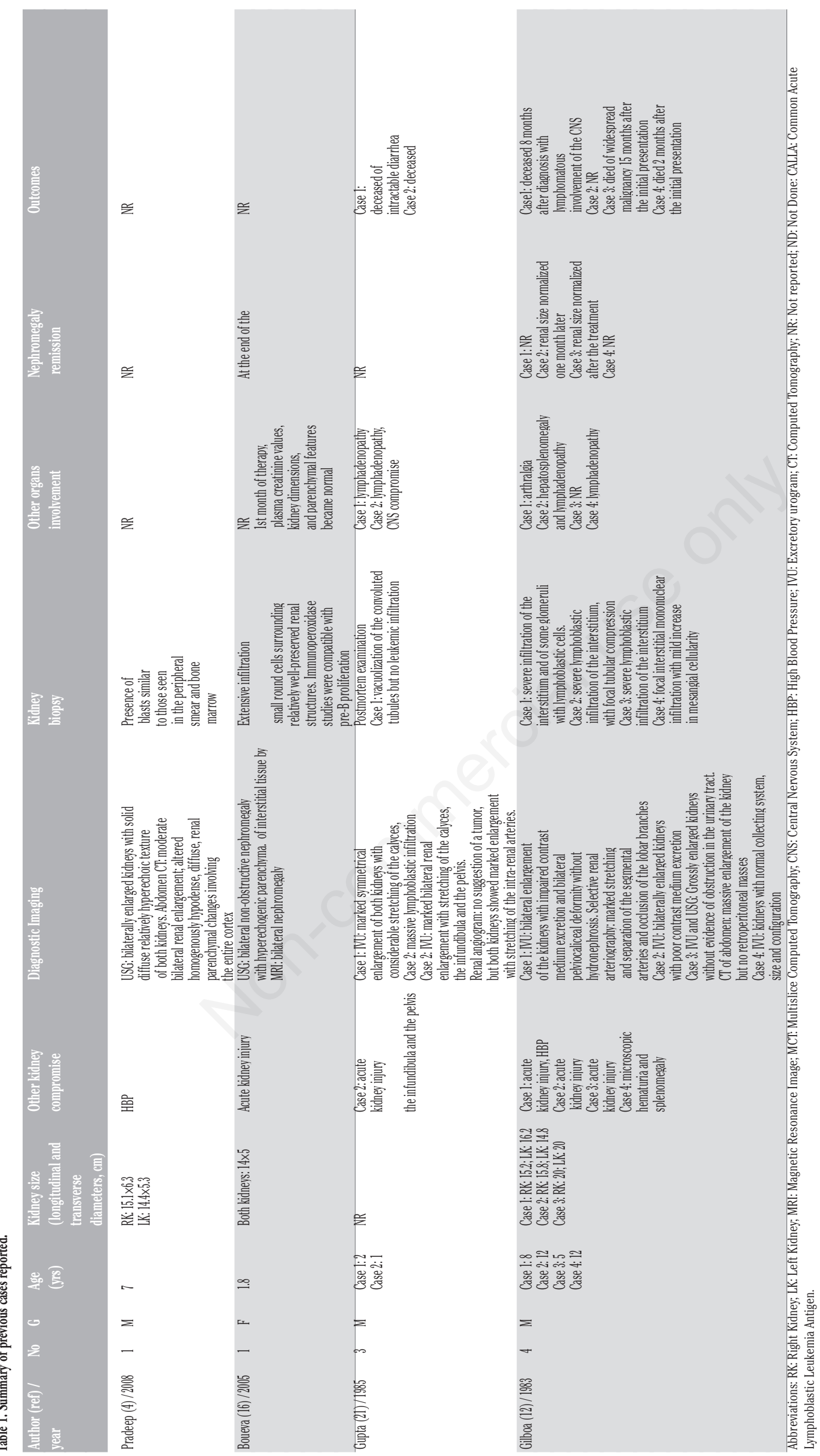


(Figure 2). The spleen and other abdominal viscera had no alterations.

Bone marrow aspirate showed $88 \%$ cellularity with $84 \%$ lymphoblasts. The immunotyping was compatible with precursor B-cell acute lymphoblastic leukemia $\left(\mathrm{CD} 19^{+}, \mathrm{CD}_{10}{ }^{+}, \mathrm{CD}_{34}{ }^{+}\right.$, weak $\mathrm{CD}^{2} 5^{+}$, CD20-/+ $(50 \%)$, weak $\mathrm{CD}^{-1} 8^{+}$, cyIgM-, cyMPO-, $\left.\mathrm{CD}^{2} 1^{+}, \mathrm{CD}^{2} 23^{+}\right)$. The cytogenetic analysis was normal, and the cerebrospinal fluid cytology was negative. A renal biopsy was not performed because the suspicion of acute leukemia was confirmed by the findings in peripheral blood and bone marrow.

In the first three days of hospitalization, blood pressure continued to be above the 99th percentile $+5 \mathrm{mmHg}$ for age, height and sex in more than $50 \%$ of measurements without treatment with corticoids. 6,7 There was no documented target organ damage (heart, retina or kidney). Treatment with amlodipine was started at $0.3 \mathrm{mg} / \mathrm{kg} / \mathrm{d}$ with adequate response.

On the third day of hospitalization, the patient started chemotherapy for intermediate-risk ALL according to ALLIC (Acute Lymphoblastic Leukemia Intercontinental) 2009 protocol (prednisolone, vincristine, daunorubicin and L-asparaginase) and received intrathecal methotrexate. The patient presented a good hematological response in peripheral blood and in bone marrow on the eighth and fifteenth day, respectively.

Kidney function was normal during the entire treatment. At the end of the induction phase of chemotherapy, a second abdominal ultrasound was performed. The size of the liver returned to its normal range and there was a decrease in the size of both kidneys.

\section{Discussion}

Acute leukemia is the most common type of cancer in pediatric patients. ${ }^{8}$ In the United States, between 2.500 to 3.500 new cases of ALL in children are diagnosed every year, with an incidence of 3.4 cases per 100.000 .1

In a meta-analysis with 33 studies and 3.084 children with leukemia, the most commonly associated signs and symptoms reported were: hepatomegaly $(64 \%)$, splenomegaly $(61 \%)$, pallor $(54 \%)$, fever $(53 \%)$, bruising $(52 \%)$, recurrent infections (49\%), fatigue (46\%), back pain (43\%), hepatosplenomegaly $(42 \%), \quad$ lymphadenopathy $(41 \%)$, bleeding tendency (38\%) and rash (35\%). ${ }^{9}$ Usually, ALL affects the bone marrow, but extramedullary involvement in liver, spleen or lymph nodes is also observed. 8,10
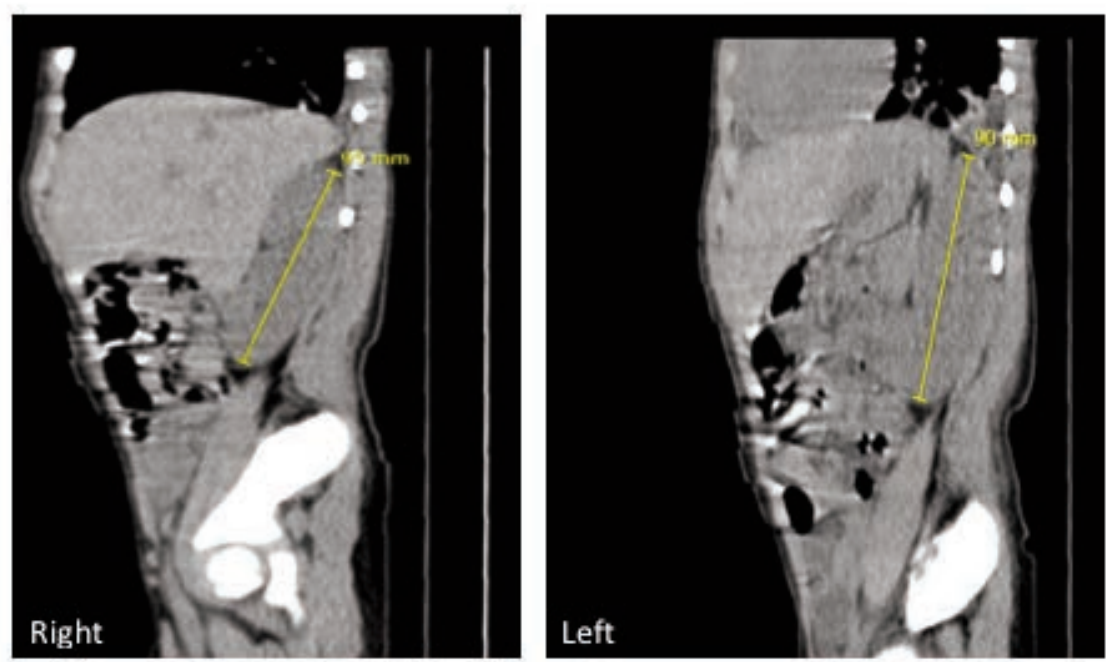

B)

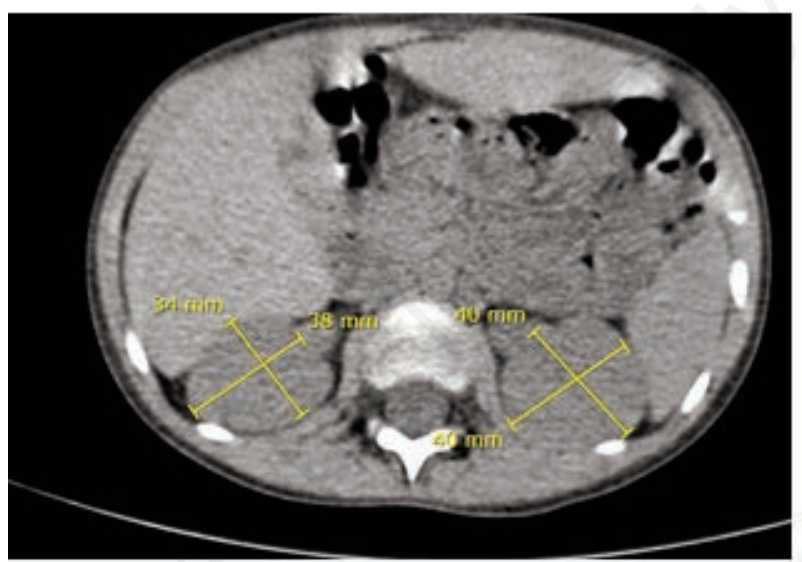

Figure 1. Computed tomography of the abdomen. Panel A: right and left kidney longitudinal diameters - Panel B: transversal diameters.

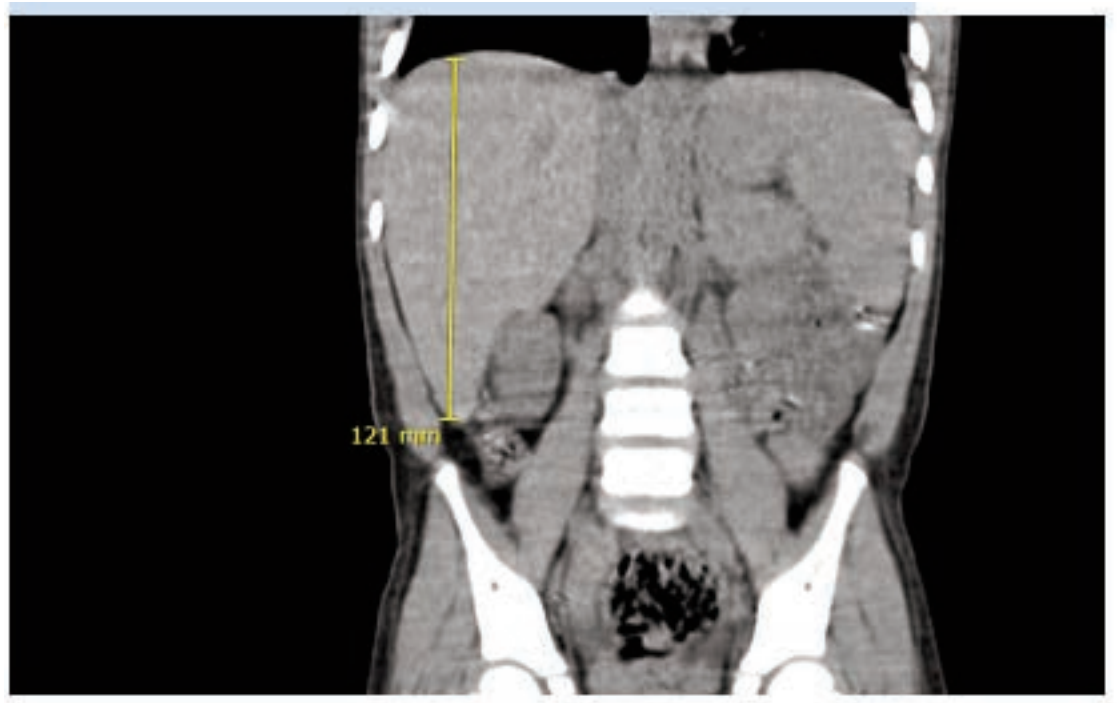

Figure 2. Computed tomography of the abdomen. Liver longitudinal diameter. 
The frequency of nephromegaly in ALL patients is variable; they have been reported to be between $2 \%$ and $24 \% .3,8,11,12$ Two etiologies of nephromegaly have been described: the first one is renal infiltration, which is more frequent in advanced stages of the disease, $5,8,13$ and it has been found in up to $50 \%$ of autopsies in pediatric patients. ${ }^{14}$ The second is hypertrophy or hyperplasia of parenchymal cells. 3,4

Renal infiltration can be diffuse or nodular. In most cases, it only involves the cortex and is symmetrical and bilateral. $4,11,13,15$ In pediatric patients, diffuse infiltration is more frequent. $12,13,16$ When renal infiltration occurs, it can be associated with the involvement of other organs such as the central nervous system, testicles and skin. ${ }^{11}$

Kidney infiltration in ALL is almost always asymptomatic and detected by the presence of a palpable abdominal mass. ${ }^{2,4,11,16}$ Abdominal ultrasound, 2 computerized axial tomography scan, 5,17 nuclear magnetic resonance (MRI) and, 8 intravenous pyelography can all be used for diagnosis. $3,12,18$

In renal ultrasound, leukemic infiltration may be suspected by the presence of enlarged kidneys with a hyperechogenic pattern, $2,5,8,16$ or by the presence of hypoechogenic nodular lesions in the kidney cortex, ${ }^{11}$ loss of corticomedullary differentiation and other abnormalities such as cystic or pyelocaliceal dilatation. ${ }^{8}$

Unlike patients with lymphoma, children with leukemia generally do not require imaging tests such as routine CT or MRI at diagnosis or follow-up. In leukemia cases, the findings in peripheral blood, bone marrow and cerebrospinal fluid, are almost always sufficient. Tests such as CT or MRI are performed when a diagnosis of related diseases or other complications is required. Therefore, it is difficult to establish the exact incidence of renal manifestations due to leukemia based on imaging tests. 15

Differential diagnoses of described changes on radiographic images include infection, lymphoma, nephroblastomatosis, cysts, angiomyolipoma or metastasis, polycystic kidney disease, renal vein thrombosis, renal diseases with organized deposits, duplication of the pelvic system, glycogen deposition diseases, Beckwith-Wiedemann syndrome and renal tumors, among others. $2,10,13,15$

In most cases reported, after the start of ALL treatment, there is an improvement in renal size, even after the first cycle of chemotherapy. $8,11,13$ Therefore, if abnormalities on radiographic images persist, a renal histopathological study may be considered.

The impact of renal leukemic infiltration on the prognosis and survival of chil- dren with ALL is uncertain. The few studies that analyze the burden of kidney infiltration on prognosis of ALL are contradictory. $10,13,17,18$

Kidney injury and HBP rarely occur in patients with ALL. Table 1 summarizes some pediatric cases reported in the literature with nephromegaly and other types of kidney compromise as primary manifestations of ALL.

HBP is usually moderate and transient, and no pharmacological treatment was required to control it in any of the reported cases. Hypertension in a patient with leukemia almost always occurs in the course of the disease. It is associated with treatment, especially long-time steroid usage, ${ }^{2}$ acute kidney injury and narrowness or occlusion of intrarenal arteries or leukemic infiltration. ${ }^{12}$ Proteinuria, hematuria or leukocyturia at diagnosis of ALL are exceptional. 12

Unlike the observations in most previous reports, in this case, hypertension occurred at the onset of the disease, required pharmacological treatment and was not associated with other comorbidities such as renal failure, hematuria, proteinuria, hyperuricemia, hypervolemia or other causes of secondary hypertension. This suggests that leukemic infiltration is not only the cause of hypertension but also the etiology of renal enlargement.

In the present case report, we hypothesize that hypertension may be related to nephromegaly. This hypothesis is supported by the improvement of HBP after the chemotherapy treatment, which allows the decrease of the dose of antihypertensive medications; the absence of white organ involvement, (which suggests a HBP of short evolution); the absence of risk factors for essential hypertension and the absence of secondary causes.

\section{Conclusions}

Nephromegaly and HBP are rare manifestations of early stages of ALL in pediatric patients. The present case report describes this unusual combination and recommends clinicians to consider malignancy as its causal factor, especially if these symptoms are accompanied by other suggestive extrarenal manifestations.

\section{References}

1. Ward E, DeSantis C, Robbins A, et al. Childhood and adolescent cancer statistics, 2014. CA Cancer J Clin
2014;64:83-103.

2. Martins A, Cairoli H, Dominguez P, et al. Nephromegaly: as unusual presentation of acute lymphoblastic leukemia in an infant. Arch Argent Pediatr. 2008;106:263-5.

3. Hann I, Lees P, Palmer MK, et al. Renal size as a prognostic factor in childhood acute lymphoblastic leukemia. Cancer 1981;48:207-9.

4. Pradeep R, Madhumathi DS, Lakshmidevi V, et al. Bilateral nephromegaly simulating wilms tumor: a rare initial manifestation of acute lymphoblastic leukemia. J Pediatr Hematol Oncol 2008;30:471-3.

5. Basker M, Scott JX, Ross B, Kirubakaran C. Renal enlargement as primary presentation of acute lymphoblastic leukaemia. Indian J Cancer 2002;39:154-6.

6. Flynn JT, Kaelber DC, Baker-Smith $\mathrm{CM}$, et al. Clinical Practice Guideline for Screening and Management of High Blood Pressure in Children and Adolescents. Pediatrics 2017;140.

7. Scholbach T, Weitzel D. Body-surfacearea related renal volume: a common normal range from birth to adulthood. Scientifica (Cairo) 2012;2012:949164.

8. Sherief LM, Azab SF, Zakaria MM, et al. Renal Presentation in Pediatric Acute Leukemia: Report of 2 Cases. Medicine (Baltimore) 2015;94:e1461.

9. Clarke RT, Van den Bruel A, Bankhead $\mathrm{C}$, et al. Clinical presentation of childhood leukaemia: a systematic review and meta-analysis. Arch Dis Child 2016;101:894-901.

10. Aguayo P, Fraser JD, St Peter SD, et al. Massive bilateral nephromegaly in acute lymphoblastic leukemia: a case report. Eur J Pediatr Surg 2009;19:3324.

11. Mantan M, Singhal KK, Sethi GR. Acute lymphoblastic leukemia: an unusual cause of nephromegaly in infancy. Indian J Pediatr 2010;77:583.

12. Gilboa N, Lum GM, Urizar RE. Early renal involvement in acute lymphoblastic leukemia and nonHodgkin's lymphoma in children. J Urol 1983;129:364-7.

13. Erdem E, Kayiran P, Ozcelik G, et al. Rare presentation of pediatric acute lymphoblastic leukemia: nephromegaly at time of diagnosis. Indian J Hematol Blood Transfus 2011:43-5.

14. Kirshbaum M, Preuss F. Leukemia. a clinical and pathologic Study of 122 fatalases in a Series of 14400 necropsies. Arch Int Med 1943;12271:777.

15. Hilmes MA, Dillman JR, Mody RJ, Strouse PJ. Pediatric renal leukemia: 